\title{
Awareness and Perceptions Regarding Evidence Based Practice Among Nurses and Midwives in Two Ugandan National Referral Hospitals: A Cross- Sectional Study
}

\section{Francine Mwitende}

Makerere University

Richard Muhindo ( $\nabla$ r.muhindo@yahoo.com )

Makerere University

\section{Research Article}

Keywords: Evidence Based Practice, awareness, perception, nurse and midwife, Uganda

Posted Date: September 22nd, 2021

DOI: https://doi.org/10.21203/rs.3.rs-881592/v1

License: (c) (1) This work is licensed under a Creative Commons Attribution 4.0 International License. Read Full License 


\section{Abstract}

Background: Integration of evidence-based practice (EBP) in nursing and midwifery increases patient satisfaction, patient safety and reduced length of hospital stay, which all contribute to better patient outcomes and improved quality of care. Little is known about awareness and perception regarding evidence-based practice among nurses and midwives in Uganda.

Objective: This study sought to assess awareness and perceptions regarding evidence-based practice among nurses and midwives.

Methods: A descriptive cross-sectional study using self-administered questionnaires was conducted among 384 nurses and midwives in Mulago and Kawempe National Referral Hospitals. The participants were recruited consecutively until when the desired sample size was obtained. Statistical Package for Social Sciences (SPSS) version 26 was used to analyze and summarize data using frequency and percentages. We performed Chi-square test to assess the factors association with awareness and perception. The independent variables with $p$-value $<0.05$ were significant.

Results: Most of the participants were female (81.8\%) and were practicing as nurses (76.2\%). The results indicated that less than a half $(46.2 \%, n=138)$ of participants were aware of EBP. Majority of the participants (96.3\%-97.7\%) felt that EBP is highly important, necessary and an obligation in nursing and midwifery practice, although only $68.0 \%$ were routinely practicing it in their practice. Awareness was associated with level of education attained $(p=0.000)$, gender $(0.001)$, going back to school after first qualification $(p=0.000)$, access to learning resources at work place $(p=0.000)$ and attendance of continuous medical education $(p=0.000)$, whereas, perception was associated with attendance of continuous medical education (0.017).

Conclusion: Data show that nurses and midwives' awareness regarding evidence-based practice was low in this study, however, they possessed positive perceptions regarding evidence-based practice. Moreover, only about two-thirds were practicing EBPs. Therefore, I recommend increased calls for interventions to sensitize nurses and midwives about EBP like continuous medical education (CME) and Continuous professional development (CPD) or refreshing training

\section{Background}

Over the decades, there has been increased calls for evidence-based practice (EBP) in nursing and midwifery care [1-4]. The drivers for evidence based practice have largely been due to; increased demand to improve patient care, involvement of patients in their own care, need for individualized care, accountability, cost-effectiveness, efficiency, professional advancement and esteem[5, 6]. Available evidence shows that in settings where nurses and midwives integrate EBP, there is increased patient satisfaction, quality of healthcare, patient safety and reduced length of hospital stay; all that contribute to better patient outcomes [7-10]. It is well documented that evidence-based nursing and midwifery practice is associated with improved patient outcome and quality of care [7, 8]. BM Melnyk found that patient 
outcomes were improved by $28 \%$ when clinical care was based on evidence rather than traditional practices[11].

Awareness and perceptions of nurses and midwives regarding EBP may play a big role in adoption and implementation of evidence based practice in nursing and midwifery practice. However, Available evidence shows that lack of awareness is one major barrier to EBP implementation [12-15]. The study done in United states found that nurses have heard of EBP but little have knowledge/skills as evidenced by their low scores (mean $=4.67, \mathrm{SD}=0.98$ ) [16]. Gerrish and Clayton (2004) found that nurses in United Kingdom know EBP concept and its impact in nursing practice, but lack of time to access sources of evidence found to be a major barrier toward implementation of EBP [17]. Review of literature showed that nurses who hold strong perception that EBP improves quality of care and patient outcome reported high extent of implementing EBP in their practice $[7,8,16,18]$. The study done in Australia showed that $87.7 \%$ of the nurses were enthusiastic about using research evidence to improve nursing practice; whereas 82.5 $\%$ felt confident about changing hospital policy if there were well-defined processes to do so [19].

Nurses and midwives contribute to the highest proportion of healthcare professionals that frequently offer services to patients in most healthcare settings [20]. In Uganda, the statistics show that nurses and midwives make $60 \%$ of the total healthcare professionals [21]. Studies show that evidence-based nursing and midwifery practice is associated with improved patient outcome and quality of care [7, 8]. However, little is known about EBP among nurses and midwives in Uganda. Knowing nurses and midwives awareness and perception regarding EBP in Uganda will help different stakeholders to make informed decisions and interventions that aim to improving the overall quality of care in the healthcare settings. Therefore, the purpose of this study was to assess awareness and perceptions regarding EBP among nurses and midwives in Uganda

\section{Method}

\section{Study design and setting}

This_cross-sectional study utilizing quantitative methods was conducted at Mulago National Referral Hospital (MNRH) and Kawempe National Referral hospitals (KNRH) in Kampala, Uganda from January to March 2020. MNRH is a specialized teaching hospital mainly for surgery and pediatrics. It had a total number of 379 nurses and midwives [22]. MNRH is located on Mulago Hill in the northern part of Kampala city immediately west of the Makerere University College of Health Sciences. KNRH located immediately north and north-east of MNRH about 5 kilometers. It had a total number of 181 nurses and midwives [23]. KNRH is also a teaching hospital mainly for obstetrics and gynecology.

\section{Study population}

The study population consisted of all nurses and midwives who were certified, licensed and registered with nursing and midwifery national council, working at MNRH and KNRH. According to data from human 
resource and nursing officer records, these hospitals had a total of 560 nurses and midwives [24, 25]. The participants had attained different levels of education varying from certificate to degree.

\section{Inclusion and exclusion criteria}

This study included both male and female nurses and midwives aged 21 to 62 years old. Pretested selfadministered questionnaire was used to collect subjective data on awareness and perception regarding EBP. Nurses and midwives who were busy at the station were excluded in the study.

\section{Sample size determination}

The sample size for this study was determined by Lisle Kish (1965) formula:

$n=\frac{Z \alpha^{2} p(1-p)}{d^{2}}$

Where: $n=$ sample size

$Z a=$ value of standard normal distribution of $95 \%$ corresponding to confidence level of 1.96

$p=$ population proportion (assumed to be $50 \%$ ).

$d=$ the maximum allowable deviation/ marginal of error which is equal to $5 \%$

Therefore, this formula gave is a total sample size of 384 participants.

\section{Sampling techniques}

Random sampling method was used to select two hospitals in Uganda. Consecutive sampling method was employed to recruit the study participants to obtain a sample size of 384 . The study participants were first grouped according to the unit or ward where they worked in. These groups were referred as clusters. The participants then well selected consecutively from each cluster until when the desired sample size was obtained. This was performed at each hospital (MNRH and KNRH).

\section{Data collection instrument}

The data were collected using pre-tested questionnaire consisted with questions which developed by the principal investigator according to study objectives. The questionnaire had total of 25 questions arranged in four sections: Section 1 had with five questions about socio-demographic characteristics of participants such as gender, age, profession, level of education attained and years of working experience. 
Section 2 had six questions about awareness regarding EBP which include whether they have had about EBP, their sources of information and their current sources of evidence for their practice. Section $3 \mathrm{had}$ five questions to assess perception, which were structured in form of Likert scale ranging from strongly agree, agree, disagree and strongly disagree. Those questions included whether EBP is highly important, an obligation and necessary to provide evidence based care and whether nurses and midwives practice EBP. Section 4 contained eight questions about factors associated with awareness and perceptions. Those questions included whether they have gone back to school after their first qualification, have attended $\mathrm{CPD}$ or $\mathrm{CME}$, have had a specific training on EBP and if they have access to learning resources at work place.

\section{Validity and reliability}

The questionnaire was pretested among 10 nursing students from Makerere University, department of nursing, who were already in practice from different institution. These students had Diploma in nursing and midwifery, and they had come back to advance their career in nursing to obtain a bachelor degree. Ambiguity and grammar errors in some items were identified and corrected to ensure the clarity. Also the questionnaire was presented to my research supervisor to ensure its adequacy in measuring the intended concept.

\section{Data collection procedure}

Data were collected from Mulago and Kawempe national referral hospitals from January to March 2020 among nurses and midwives who were at work during data collection period. The study was approved by research and ethics committee (REC) of the school of health sciences, Makerere University and by the respective institutional review boards of MNRH and $\mathrm{KNRH}$ ). The study was conducted according to the declaration of Helsinki and good clinical practices. All participants provided a written informed consent prior to participating in the study. The principal investigator trained other two nursing students to assist with data collection. After being introduced by the in-charge, the researcher informed the participants about the study and requested to voluntarily sign the consent. Those who signed consent were able to access self-administered questionnaires following consecutive sampling method. The questionnaires were distributed among the study participants during their shift and filled them at their convenient time and return them the same day or on next shift. The total of 384 questionnaires were distributed. The questionnaires completeness was checked.

\section{Data analysis}

The primary outcomes of this study were awareness and perception regarding EBP, which were summarized and reported as frequencies and proportions for categorical data or median (range) and mean (standard deviation) for numerical data depending on the distribution (normal or non-normal 
distribution). Data analysis was performed using by SPSS version 26. Chi-Square test and binary logistic regression were done to study the relationship between the dependent and independent variables. P-value of $<0.05$ was statistically significant. For the case of perception, the four items on Likert scale were computed into one item and the mean score was calculated which was four. A score of three and above was considered as positive perception, while the score less than three considered as poor perception.

\section{Results}

A total of 384 questionnaires were distributed and only 303 complete questionnaires were returned, contributing to response rate of $78.9 \%$. Table 1 summarizes the socio-demographic characteristics of the participants. Majority $(81.8 \%, \mathrm{n}=248)$ of the participants were female, practicing as nurses $(76.2 \%)$ and the median age was 32 (ranging from 21-62) years whereas the median years of working experience was 8 (ranging from 0-43) years.

\section{Nurses and midwives' awareness with regards to EBP}

Overall, $46.2 \%(n=138)$ of the participants had heard about EBP. Figure 1 shows the sources of information (where they heard about EBP) from, whereas figure 2 presents their current sources of evidence for their practice.

\section{Nurses and midwives' perception regarding EBP.}

Perception was studied among 138 participants who were aware of EBP (Table 2). Significant number of the participants (97\%) expressed positive perception toward EBP whereas only $3 \%$ had poor perception. However, over half (68\%) were practicing EBP routinely

\section{Factors associated with nurses and midwives' awareness regarding EBP.}

Level of education $(p<0.000)$, qualification upgrade $(p<0.000)$, and access to learning resources $(p<0.000)$ were significantly associated with EBP Table 2.

\section{Factors associated with nurses and midwives' perceptions regarding EPB}

Attendance of continuous medical education (CME) and continuous professional development (CPD) P= 0.001 was associated with nurses and midwives' perception Table 3. 


\section{Discussion}

The main objective of this study was to assess nurses and midwives' awareness and perceptions regarding EBP. The findings showed that less than a half (46.2\%) were aware of EBP. Most nurses and midwives (over $97 \%$ ) possessed positive perceptions regarding EBP, however only $68 \%$ routinely practice EBP. Level of education attained, going back to school after first qualification, gender, access to learning resources and attendance of CME/CPD were significantly associated with awareness and perceptions.

\section{Awareness of nurses and midwives with regards to EBP}

From this study, less than half (46.2\%) of the participants were aware of EBP. These findings were lower than that in the study done in Oman [7], Ethiopia [10], and Poland [26] where 63.3\% v, 62.6\% and 66.6\% were aware of EBP, respectively. All these studies were conducted in teaching hospitals with specialized experience, which is similar to our study. This inconsistence in study findings may be due to the difference in participant's characteristics, especially the levels of education attained. Most participants were bachelor's degree holder whereas in our study, the majority were diploma and enrolled nurses and midwives. It may also be due to the numbers of exposure to the concept and implementation of EBP in Oman, Poland and Ethiopia, which is higher than here in Uganda.

The finding showed multiple sources of awareness on EBP, where over a half $(60 \%)$ heard about EBP from CME and CPD attended. Another significant number heard about EBP from learning institution and reading professional textbooks. About the current sources of evidence for nursing and midwifery practice, the majority of participant rely their practice on personal expertise, review of literature and clinical guidelines. It is encouraging that some nurses and midwives are relying on scientific evidence, however the habit of try and error still exists in nursing and midwifery practice. Early studies done in United Kingdom, Australia and Iran found similar findings and the majority of nurses and midwives are progressively adopting evidence from disciplined research other than traditional sources $[17,27,28]$.

The findings from this study also revealed some factors which could preventing nurses and midwives to be aware of EBP. These factors include the level of education attained, going back to school after first qualification, gender and access to learning resources. Prior study found that nurses and midwives who has a bachelor's degree and above were more familiar with the concept of EBP[29, 30]. In Uganda, the concept of EBP is included in the curriculum of undergraduate nursing and midwifery studies. Moreover, some health facilities provide specific trainings about EBP, which can be beneficial to those who did not attain bachelor's degree. Furthermore, the studies done in USA, Kenya and Egypt found that limited access to learning resources prevent nurses and midwives from knowing and implementing EBP [30-32]. This highlight the need of interventions of availing accepted learning materials available at their place of work such as libraries, standard operating procedures (SOPs) and printed guidelines. 


\section{Perceptions with regards to EBP among nurses and midwives}

Nurses and midwives expressed positive perception about EBP in Nursing and Midwifery practices where most participants agreed that EBP is highly important (97.0\%), it is necessary to provide care based on evidence $(97.7 \%)$ and it is an obligation for every nurse and midwife to provide evidence-based care (96.3\%). However $68.0 \%$ routinely practice EBP. This means that if nurses and midwives are given opportunity to know much about EBP, they will implement it in their practice. These findings from this study were consistent with the studies done among nurses and midwives from Oman[7], Iran[27], USA[11] and Australia[16, 19, 33]. These studies found that nurses and midwives' perceptions and beliefs were strong and positive. Across the world, nurses and midwives expressed strong and positive perceptions regarding EBP $[10,27,34]$, although their knowledge regarding it remains low leading to inconsistence in implementation of EBP in nursing and midwifery practices[12, 35]. In this study, attendance of CME/CPD was a major factor associated with nurses and midwives perceptions. It is therefore recommended that opportunities toward CME/CPD should be extended to nurses and midwives during their clinical practice.

\section{Limitations}

Since the study participants were selected by Use of consecutive sampling method, there is a possibility of selection bias. However, this sampling method was the best considering the nature of the study. Secondly, the study used self-administered questionnaire, which reduced the response rate to $78.9 \%$, however this response rate is accepted and sufficient in quantitative research.

\section{Conclusion}

The overall nurses and midwives' awareness regarding EBP was low. The nurses and midwives possessed positive perceptions regarding EBP. The factors associated with awareness regarding EBP included level of education attained, gender, going back to school after first qualification and access to learning resources at workplace whereas perception was only associated with attendance of CME/CPD

\section{Abbreviations}

CME: Continuous Medical Education; CPD: Continuous Professional Development; EBP: Evidence Based Practice, MNRH: Mulago National Referral Hospital and KNRH: Kawempe National Referral Hospital, SOPs: Standard Operating Procedures.

\section{Declarations}


We would like to confirm that all methods were performed in accordance with the relevant guidelines and regulations for this particular journal.

\section{Ethics approval and consent to participate.}

Ethical approval to conduct this study was sought from the research and ethics committee of the school of health sciences, Makerere University, Mulago and Kawempe research and ethical committee. The purpose, benefits, and procedure of the study were clearly explained to the participants before conducting the study. Participants were requested to voluntarily sign written consent forms as proof of consent. Privacy and confidentiality were ensured throughout the study.

\section{Consent for publication}

This is not applicable.

\section{Availability of data and materials}

The dataset upon which the findings and conclusions of this study are based can be obtained from the corresponding author under reasonable request.

\section{Competing interests}

The authors declare that they have no conflicts of interest.

\section{Funding}

The study was funded by Mastercard Foundation Scholars Program at Makerere University

\section{Authors' contributions}

The manuscript was drafted and reviewed by all the authors for important intellectual content. The authors read and approved the final manuscript.

\section{Acknowledgement}

Author wishes to express sincere gratitude to nurses and midwives from Mulago and Kawempe National Referral Hospitals who volunteered to participate in this study. I would like to thank Olum Ronald, for his 
mentorship and assistance during manuscript writing. Am also honored to send my sincere thanks to my mentors Dr. TUMUSIIME Gerald and Ms. Elizabeth Ayebare for social and psychological support.

\section{References}

1. Bonell, C.J.J.o.A.N., Evidence-based nursing: a stereotyped view of quantitative and experimental research could work against professional autonomy and authority. 1999. 30(1): p. 18-23.

2. Estabrooks, C.A.J.C.J.o.N.R.A., Will evidence-based nursing practice make practice perfect? 1998. 30(1).

3. Gurzick, M. and K.S.J.J.o.P.N. Kesten, The impact of clinical nurse specialists on clinical pathways in the application of evidence-based practice. 2010. 26(1): p. 42-48.

4. Mitchell, G.J.J.N.S.Q., Evidence-based practice: Critique and alternative view. 1999. 12(1): p. 30-35.

5. Jylhä, V., et al., Facilitating evidence-based practice in nursing and midwifery in the WHO European Region. 2017.

6. Melnyk, B.M. and E. Fineout-Overholt, Evidence-based practice in nursing \& healthcare: $A$ guide to best practice. 2011: Lippincott Williams \& Wilkins.

7. Ammouri, A.A., et al., Evidence-Based Practice: Knowledge, attitudes, practice and perceived barriers among nurses in Oman. Sultan Qaboos University medical journal, 2014. 14(4): p. e537-e545.

8. BernadetteMazurekMelnyk, R., PhD,CPNP/NPP,FAAN,FNAP,EllenFineout-

Overholt,RN,PhD,NancyFischbeckFeinstein,RN-C,PhD, HongLi,RN,PhD,LeighSmall,RN-

CS,PhD,PNP,LarryWilcox,RN,MS,NP-BC,RachelKraus,RN,MS,PNP, Nurses' Perceived Knowledge, Beliefs, Skills, and Needs Regarding Evidence-Based Practice: Implications for Accelerating the Paradigm Shift. 2004.

9. Calderón2, C.C.a.J., Evidence-based practice in Chile 2018.

10. Hoyiso, D., et al., Evidence based nursing practice and associated factors among nurses working in Jimma zone public hospitals, Southwest Ethiopia. 2018. 10(5): p. 47-53.

11. Bernadette Mazurek Melnyk, R., PhD,CPNP/NPP,FAAN,FNAP,EllenFineoutOverholt,RN,PhD,NancyFischbeckFeinstein,RN-C,PhD, HongLi,RN,PhD,LeighSmall,RNCS,PhD,PNP,LarryWilcox,RN,MS,NP-BC,RachelKraus,RN,MS,PNP, Nurses' Perceived Knowledge, Beliefs, Skills, and Needs Regarding Evidence-Based Practice: Implications for Accelerating the Paradigm Shift. 2004.

12. Elham Azmoude1, M.A., Faezeh dehghAni3, Midwives' Attitude and Barriers of Evidence Based Practice in Maternity Care. 2018.

13. Funk, S.G., E.M. Tornquist, and M.T.J.N.C.o.N.A. Champagne, Barriers and facilitators of research utilization. 1995. 30(3): p. 395-407.

14. Khammarnia, M., et al., Barriers to implementation of evidence based practice in zahedan teaching hospitals, iran, 2014. Nursing research and practice, 2015. 2015: p. 357140-357140. 
15. Review, B.A.W.E.-B.P.A.N.i.L.-a.M.-I.C.A.S., Barriers Associated With Evidence- Based Practice Among Nurses in Low- and Middle-Income Countries: A Systematic Review. 2019.

16. Lehman, M.L.K.K., Nurses' perceptions of evidence-based nursing practice. 2007.

17. Gerrish, K. and J.J.J.o.n.m. Clayton, Promoting evidence-based practice: an organizational approach. 2004. 12(2): p. 114-123.

18. Pereira, F., M. Salvi, and H. Verloo, Beliefs, Knowledge, Implementation, and Integration of EvidenceBased Practice Among Primary Health Care Providers: Protocol for a Scoping Review. JMIR research protocols, 2017. 6(8): p. e148-e148.

19. Sherriff, K.L., M. Wallis, and W.J.I.j.o.n.p. Chaboyer, Nurses' attitudes to and perceptions of knowledge and skills regarding evidence-based practice. 2007. 13(6): p. 363-369.

20. Spero, J.C., P.A. McQuide, and R.J.H.R.f.H. Matte, Tracking and monitoring the health workforce: a new human resources information system (HRIS) in Uganda. 2011. 9(1): p. 6.

21. Francis, P., Peter,Robert, Henry, Katumba and Augustine, HEALTH WORKFORCE MIGRATION: A CASE STUDY OF GENERAL PRACTITIONERS IN UGANDA. 2017.

22. Human resource records, Total employees at Mulago national referral hospital. 2019.

23. Senior nursing officer records, The number of Nurses and Midwives at Kawempe national referral hospital 2019.

24. records, H.r., nurses in Mulago hospital. 2019.

25. records, H.r., Nurses and Midwives at Kawempe hospital. 2019.

26. Belowska, J., et al. KNOWLEDGE OF AND ATTITUDES TOWARDS EVIDENCE-BASED PRACTICE AMONG MIDWIVES TAKING A SPECIALTY EXAMINATION ORGANIZED BY CENTER OF POSTGRADUATE EDUCATION FOR NURSES AND MIDWIVES. in INTED2016 Proceedings. 2016. IATED.

27. Adib-Hajbaghery, M.J.W.o.E.B.N., Evidence-based practice: Iranian nurses' perceptions. 2009. 6(2): p. 93-101.

28. Waters, D., et al., The Australian experience of nurses' preparedness for evidence-based practice. 2009. 17(4): p. 510-518.

29. Shaheen Majid, P.S.F., PhD; Brendan Luyt, PhD; Xue Zhang, MSc; Yin-Leng Theng, PhD; Yun-Ke Chang, PhD; Intan A. Mokhtar, PhD, Adopting evidence-based practice in clinical decision making: nurses' perceptions, knowledge, and barriers. 2011.

30. Barako, T.D., et al., Factors influencing application of evidence-based practice among nurses. 2012. 6(2): p. 71-77.

31. Brown, C.E., et al., Nursing practice, knowledge, attitudes and perceived barriers to evidence-based practice at an academic medical center. 2009. 65(2): p. 371-381.

32. Mohsen, M.M., N.A. Safaan, and O.M.J.A.J.o.N.R. Okby, Nurses' perceptions and barriers for adoption of evidence based practice in primary care: Bridging the gap. 2016. 4(2): p. 25-33.

33. Shafiei, E., et al., Nurses' perceptions of evidence-based practice: a quantitative study at a teaching hospital in Iran. Medical journal of the Islamic Republic of Iran, 2014. 28: p. 135-135. 
34. Correa-de-Araujo, R., Evidence-Based Practice in the United States: Challenges, Progress, and Future Directions. Health care for women international, 2016. 37(1): p. 2-22.

35. Howard, J.M.J.a.M.O., Evidence-Based Practice 2013.

\section{Tables}

Due to technical limitations, tables are only available as a download in the Supplemental Files section.

\section{Figures}

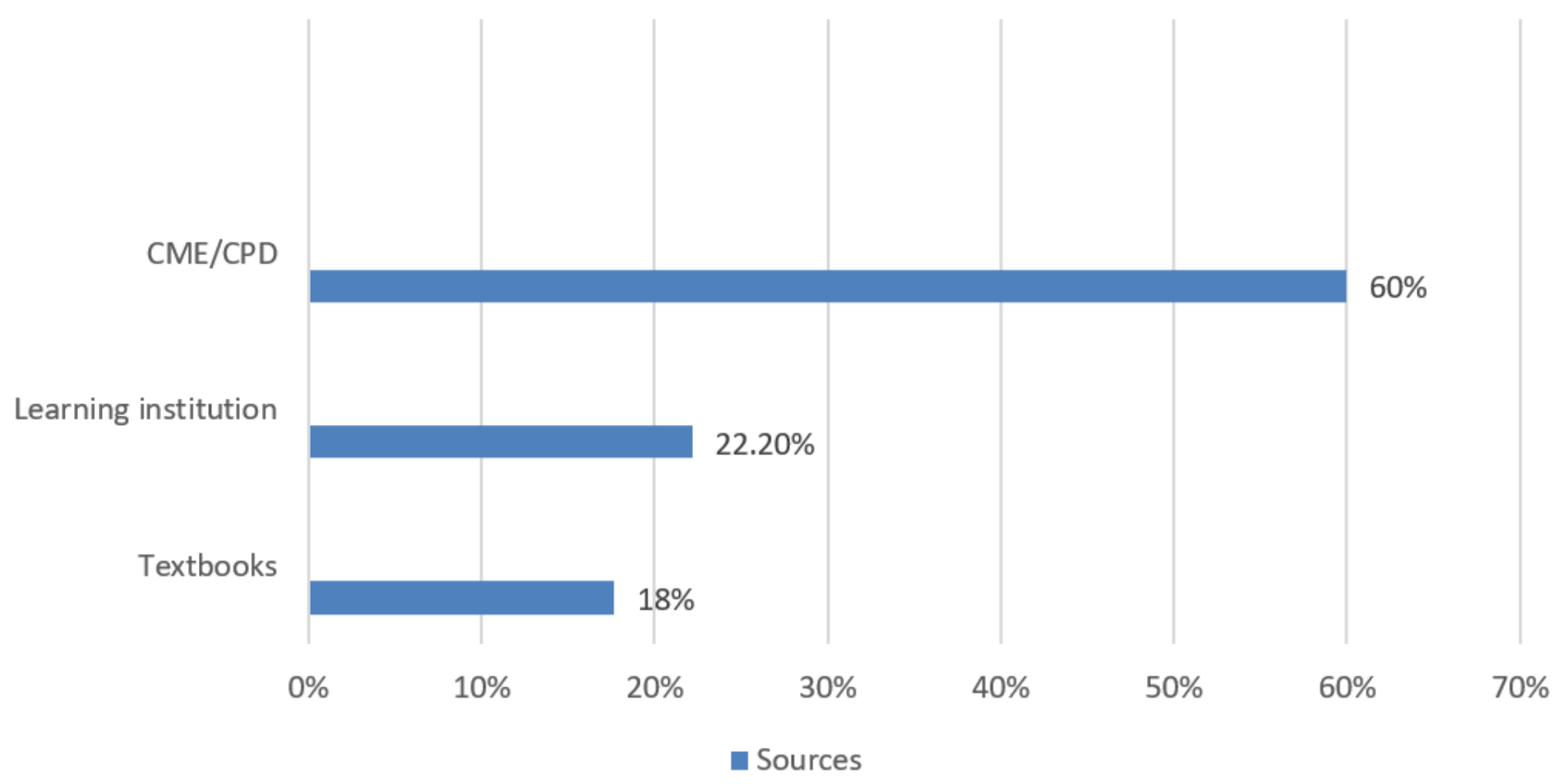

Figure 1

sources of information about EBP 


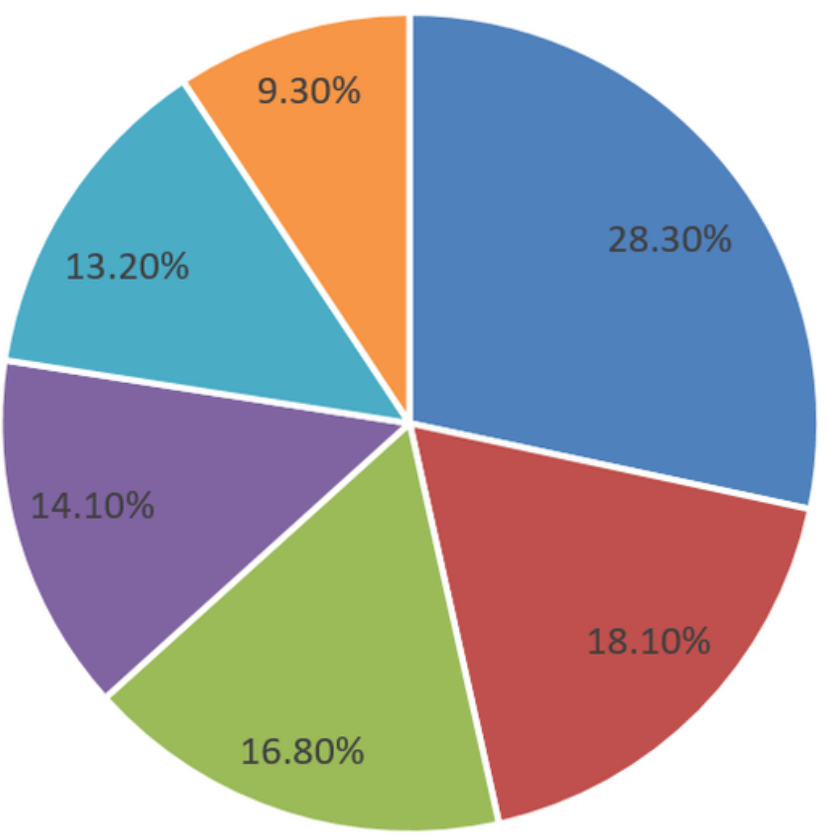

- Experience

- Literature review

- Clinival guidelines

- Google search

a consulting seniors

- Professional textbooks

Figure 2

Sources of evidence for nursing and midwifery practive

\section{Supplementary Files}

This is a list of supplementary files associated with this preprint. Click to download.

- Tables.docx 